\title{
Machbarkeit - Finanzierbarkeit - Ethik: Medizinischer Fortschritt als gesellschaftliche Herausforderung
}

\author{
1. Luzerner Trendtage Gesundheit - 22./23. Februar 2005, Luzern. Interview mit Regierungsrat M. Dürr. \\ C. Wicki
}

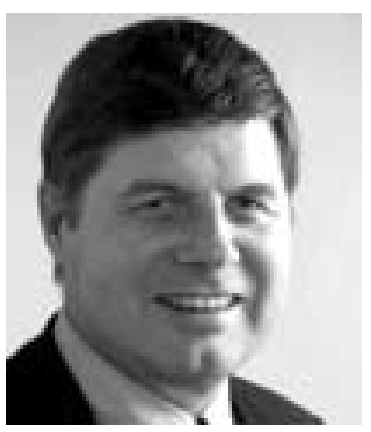

Regierungsrat Dr. Markus Dürr
Ein neuer Kongress im Februar in Luzern zur aktuellen Gesundheitsdebatte weckt Hoffnungen und Erwartungen. Der Anlass will eine Lücke im Kongressangebot schliessen und Anbieter, Nachfrager, Bezahlende, Wirtschaft sowie Forschung und Wissenschaft zusammenbringen. Mit der Wahl des dreiteiligen Themenspektrums «Machbarkeit - Finanzierbarkeit - Ethik» wird ein Diskussionsschwerpunkt aufgenommen, der mittelfristig die gesundheitspolitischen Debatten prägen dürfte. Ziel ist die Etablierung einer jährlich wiederkehrenden interdisziplinären Dialogplattform zur Zukunft der Gesundheitsversorgung.

Regierungsrat Dr. Markus Dürr, Vorsteher des Gesundheits- und Sozialdepartements des Kantons Luzern und Präsident der GDK, ausserdem Vorsitzender des Forums Gesundheit Luzern, der Trägerorganisation der Luzerner Trendtage Gesundheit, äussert sich zum konzeptionellen Umfeld der Tagung.

Die Thematik des Anlasses scheint aktueller denn je. Das ist zwar reizvoll, um einen neuen Kongress zu starten - was will man letztlich aber genau erreichen? Wie kann das Publikum konkret profitieren, was darf es erwarten?

An diesem Kongress sollen z. B. folgende Fragen diskutiert werden: Rechtfertigt der Zusatznutzen bestimmter neuer Behandlungsmethoden die Zusatzkosten? Verpassen wir eine Chance, wenn wir bestimmte Neuerungen nicht einführen oder noch zuwarten? Wo können wir ohne schlechtes Gewissen sparen? Was kann Gentherapie und was nicht? Welches sind die Chancen und Risiken der Nanotechnologie? Welche Innovatio-
Korrespondenz:

Dr. sc. nat. Christof Wicki Forum Gesundheit Luzern Horwerstrasse 87 CH-6005 Luzern Tel. 0413183797 Fax 0413183710

E-Mail: info@trendtage-gesundheit.ch Website: www.trendtage-gesundheit.ch

\section{EL U Z E N E R DT T E D T A G E} a G U U D HEIT

Die Luzerner Trendtage Gesundheit finden erstmals am 22./23. Februar 2005 im KKL Luzern statt. Organisator ist der Trägerverein Forum Gesundheit Luzern.

Anmeldeschluss ist der 18. Februar 2005.

Preise zwischen Fr. 130.- (erster Tag, reduziert) und 480.- (zwei Tage, regulär).

Das Programmheft kann von der Website des Forums Gesundheit Luzern heruntergeladen werden: www.trendtage-gesundheit.ch.

Der Verein steht allen Kreisen offen, welche die Ziele des Vereins unterstützen (Jahresbeitrag). Vereinsmitglieder erhalten Zutritt zum reduzierten Tarif.

Information, Programm und Anmeldung

Forum Gesundheit Luzern, Dr. med. Christof Wicki, Horwerstrasse 87, 6005 Luzern, Tel. 041 31837 97, Website: www.trendtage-gesundheit.ch 
nen aus der Medizintechnik oder Pharmaforschung kommen auf uns zu und welche Kostenfolgen haben sie?

Der Fokus soll dabei nicht auf die Schweiz beschränkt bleiben. Erfahrungen und Lösungsansätze aus andern Ländern wollen wir mitberücksichtigen.

Sie plädieren für eine bessere Ressourcenallokation. Sie holen dabei bewusst den Disput zwischen Rationalisierung und Rationierung aufs Tapet. Auf welcher Seite liegt die wahre Lösung? Natürlich müssen wir zuerst dort sparen, wo es am wenigsten «weh» tut. Das ist in der Regel beim Rationalisieren. Das Rationalisierungspotential ist aber irgendwann einmal ausgeschöpft. Und dann kommt man nicht um die Rationierung herum. Rationalisieren vermag deshalb die Rationierung höchstens zeitlich hinauszuschieben, nicht aber zu verhindern.

Sie vertreten die Meinung, dass eine effiziente Ressourcenallokation nicht allein der Ärzteschaft aufgebürdet werden kann, also dass weitere Entscheidungsträger mitreden sollten. Ist mit dieser Absicht nicht ein gesellschaftspolitisches Hickhack vorprogrammiert?

Natürlich ist es ein schwieriger Weg. Aber gleichzeitig auch der einzig richtige. Einerseits ist es unfair gegenüber der Ärzteschaft, wenn sie allein darüber entscheiden muss, welche Leistungen welchen Patienten nicht mehr angeboten werden. Die Politik macht es sich allzu einfach, wenn sie mit dem Budget zwar Rationierung fordert, aber keine näheren Ausführungen dazu macht. Und anderseits wollen und sollen in diesen wichtigen Fragen auch verschiedene andere Betroffene mitreden. Wenn der Arzt allein entscheiden muss, werden je nach Druck der Angehörigen, der zur Verfügung stehenden Möglich- keiten und der Einstellung des Arztes für analoge Fälle unterschiedliche Entscheide getroffen. Rationierung muss deshalb wenn immer möglich nach fairen, transparenten und demokratisch festgelegten Regeln passieren. Heute ist das Gegenteil der Fall.

Die Luzerner Trendtage wollen ethische Aspekte gleichberechtigt in die Diskussionen einbringen. Wie kann in einem knallharten Business, dem sich der Gesundheitsmarkt nüchtern betrachtet immer mehr annähert, den ethischen Anliegen der notwendige Spielraum und das erforderliche Stimmengewicht verschafft werden?

Ich sehe das nicht so pessimistisch, sondern bin im Gegenteil überzeugt davon, dass sich die Wirtschaft sehr wohl bewusst ist, dass ethische Aspekte beim Gesundheitsmarkt eine sehr grosse und wahrscheinlich eine immer grössere Rolle spielen. Sie hat deshalb durchaus ein Interesse, auch ethische Aspekte zusammen mit ihren Kunden zu diskutieren.

Sie sind Präsident des Trägervereins und Präsident des Advisory Boards der Tagung. Warum dieses persönliche Engagement?

Als Gesundheitsdirektor und Präsident der Gesundheitsdirektorenkonferenz bin ich täglich mit dem Spannungsfeld zwischen Machbarkeit, Finanzierbarkeit und Ethik konfrontiert. Die Schere zwischen Machbarkeit und Finanzierbarkeit öffnet sich immer mehr. Und während einerseits laufend medizinische Innovationen präsentiert und gelobt werden, wird anderseits lautstark gefordert, dass die Kosten gesenkt werden müssen. Ich plädiere deshalb schon seit längerem für einen offenen und ehrlichen Umgang mit dieser Problematik. Die Trendtage Gesundheit können dazu positive Impulse geben und die Diskussionen versachlichen. 